\title{
DNA approach for password conversion generator
}

\begin{abstract}
The evolution of an information technology has been expended and growth rapidly since last decade, especially in the era of an internet web technology, such as, e-commence, ebusiness or e-payment or e-shopping and more. The evolution of an internet web technology has made the transmission of the data or information over the web is more comprehensive. Thus, the data or information is easy to hack, crack or spy by the unauthorized persons over the network. This paper proposed a technique of cryptography to make the data or information to be more secure during transmission over the internet technology based on the DNA Stenography with the Finite State Machine (Mealy Machine) theory. This proposed algorithm is able to securing the data or information at least 3 levels of combinations for the password conversion.
\end{abstract}

Keyword: Automata; Cryptographic; DNA; E-commence; Security 